\title{
Erratum to: A highly sensitive chemical gas detecting transistor based on highly crystalline CVD-grown $\mathrm{MoSe}_{2}$ films
}

Jongyeol Baek ${ }^{1, \S}$, Demin Yin ${ }^{2, \S}, \mathrm{Na} \mathrm{Liu}^{1, \S}$, Inturu Omkaram ${ }^{1}$, Chulseung Jung ${ }^{1}$, Healin $\mathrm{Im}^{1}$, Seongin Hong ${ }^{1}$, Seung Min $\mathrm{Kim}^{3}$, Young Ki Hong ${ }^{1}$, Jaehyun $\mathrm{Hur}^{4}(\varangle)$, Youngki Yoon ${ }^{2}(\varangle)$, and Sunkook $\mathrm{Kim}^{1}(\varangle)$

${ }^{1}$ School of Advanced Materials Science \& Engineering, Sungkyunkwan University, Suwon 16419, Republic of Korea

${ }^{2}$ Department of Electrical and Computer Engineering \& Waterloo Institute for Nanotechnology (WIN), University of Waterloo, Waterloo, ON N2L 3G1, Canada

${ }^{3}$ Institute of Advanced Composite Materials, Korea Institute of Science and Technology (KIST), Jeonbuk 565-905, Republic of Korea

${ }^{4}$ Department of Chemical and Biological Engineering, Gachon University, Seongnam-si, Gyeonggi 13120, Republic of Korea

${ }^{\S}$ These authors contributed equally to this work.

C) Tsinghua University Press and Springer-Verlag Berlin Heidelberg 2017

\section{Erratum to}

Nano Research 2017, 10(6): 1861-1871

DOI 10.1007/s12274-016-1291-7

The first affiliation of the authors in the original version of this article was unfortunately wrongly written on page 1861 and the first page of the ESM, instead of Multi-Functional Nano/Bio Electronics Lab., Kyung Hee University, Gyeonggi 446-701, Republic of Korea.

It should read

${ }^{1}$ School of Advanced Materials Science \& Engineering, Sungkyunkwan University, Suwon 16419, Republic of Korea

The online version of the original article can be found at

http://dx.doi.org/10.1007/s12274-016-1291-7

Address correspondence to Jaehyun Hur, hjhhj76@gmail.com; Youngki Yoon, youngki.yoon@uwaterloo.ca; Sunkook Kim, inte10616@gmail.com 